\title{
Safety and efficacy of the Pipeline Embolization Device in 100 small intracranial aneurysms
}

\author{
Nohra Chalouhi, MD, ${ }^{1}$ Mario Zanaty, MD, ${ }^{1}$ Alex Whiting, MD, ${ }^{1}$ Steven Yang, MS, ${ }^{1}$ \\ Stavropoula Tjoumakaris, MD, ${ }^{1}$ David Hasan, MD, ${ }^{2}$ Robert M. Starke, MD, ${ }^{3}$ Shannon Hann, MD, ${ }^{1}$ \\ Christine Hammer, MD, ${ }^{1}$ David Kung, MD, ${ }^{1}$ Robert Rosenwasser, MD, ${ }^{1}$ and Pascal Jabbour, MD1
}

\begin{abstract}
1Department of Neurological Surgery, Thomas Jefferson University and Jefferson Hospital for Neuroscience, Philadelphia, Pennsylvania; ${ }^{2}$ Department of Neurosurgery, University of lowa, lowa City, lowa; and ${ }^{3}$ Department of Neurological Surgery, University of Virginia, Charlottesville, Virginia
\end{abstract}

OBJECT Flow diverters are increasingly used for treatment of intracranial aneurysms. In most series, the Pipeline Embolization Device (PED) was used for the treatment of large, giant, complex, and fusiform aneurysms. Little is known about the use of the PED in small aneurysms. The purpose of this study was to assess the safety and efficacy of the PED in small aneurysms $(\leq 7 \mathrm{~mm})$.

METHODS A total of 100 consecutive patients were treated with the PED at the authors' institution between May 2011 and September 2013. Data on procedural safety and efficacy were retrospectively collected.

RESULTS The mean aneurysm size was $5.2 \pm 1.5 \mathrm{~mm}$. Seven patients $(7 \%)$ had sustained a subarachnoid hemorrhage. All except 5 aneurysms (95\%) arose from the anterior circulation. The number of PEDs used was 1.2 per aneurysm. Symptomatic procedure-related complications occurred in 3 patients (3\%): 1 distal parenchymal hemorrhage that was managed conservatively and 2 ischemic events. At the latest follow-up (mean 6.3 months), 54 (72\%) aneurysms were completely occluded (100\%), $10(13 \%)$ were nearly completely occluded $(\geq 90 \%)$, and $11(15 \%)$ were incompletely occluded (<90\%). Six aneurysms (8\%) required further treatment. Increasing aneurysm size (OR 3.8, 95\% Cl 0.99-14; p $=0.05$ ) predicted retreatment. All patients achieved a favorable outcome (modified Rankin Scale Score 0-2) at follow-up.

CONCLUSIONS In this study, treatment of small aneurysms with the PED was associated with low complication rates and high aneurysm occlusion rates. These findings suggest that the PED is a safe and effective alternative to conventional endovascular techniques for small aneurysms. Randomized trials with long-term follow-up are necessary to determine the optimal treatment that leads to the highest rate of obliteration and the best clinical outcomes.

http://thejns.org/doi/abs/10.3171/2014.12.JNS14411

KEY WORDS intracranial aneurysm; flow diversion; Pipeline Embolization Device; small aneurysm; vascular disorders

$\mathrm{F}$ Low diverters are now routinely used at several institutions for treatment of an expanding population of intracranial aneurysms. . $, 8,13,18^{\text {The Pipeline Embo- }}$ lization Device (PED) is a flow diverter that has received significant attention in the recent literature. ${ }^{7,17,18}$ The PED was approved by the FDA in 2011 for treatment of large and giant wide-necked aneurysms of the internal carotid artery (ICA). In most series the PED was used for the treatment of large, giant, complex, and fusiform aneurysms, with favorable results. Many investigators, however, remain wary of flow diverters, citing the lack of long-term safety data as well as the risk of distal parenchymal hemorrhage and delayed aneurysm rupture.

Currently, there are few data on the use of the PED in small aneurysms amenable to conventional endovascular techniques. . $14,19,20$ Therefore, the safety and efficacy of the device in this subgroup remains unknown. We present the results of the largest series of small intracranial aneurysms treated with the PED to date.

\section{Methods}

\section{Study Design}

This is a retrospective review of a single-center experience with use of the PED to treat small intracranial aneurysms. The study protocol was approved by the Thomas Jefferson University Institutional Review Board.

ABBREVIATIONS ICA = internal carotid artery; $\mathrm{MCA}=$ middle cerebral artery; $\mathrm{mRS}=$ modified Rankin Scale; $\mathrm{PED}=$ Pipeline Embolization Device; SAH = subarachnoid hemorrhage.

SUBMITTED February 22, 2014. ACCEPTED December 2, 2014.

INCLUDE WHEN CITING Published online January 30, 2015; DOI: 10.3171/2014.12.JNS14411.

DISCLOSURE Drs. Jabbour and Tjoumakaris are consultants for Covidien, and Dr. Tjoumakaris is also a consultant for Stryker. 


\section{Settings, Participants, and Study Size}

Small aneurysms were defined as aneurysms $\leq 7 \mathrm{~mm}$ in largest diameter. A total of 100 consecutive patients with small aneurysms were treated with the PED at our institution between May 2011 and September 2013. Some findings in patients included in this study have been reported in a previous paper. ${ }^{11}$ Currently, the PED is a first-line option for large, giant, and fusiform aneurysms at our institution. As we have become more comfortable with flow diverters, we currently treat most unruptured aneurysms (both morphologically simple and complex), except bifurcation and anterior communicating artery aneurysms, with the PED. In several cases, aneurysms treated with the PED are amenable to conventional strategies such as clipping or coiling. We generally avoid using the PED for ruptured aneurysms and reserve it for aneurysms that are strictly unamenable to conventional endovascular and surgical techniques (such as blister aneurysms).

\section{Implantation Procedure}

Patients undergoing PED therapy for unruptured aneurysms received $75 \mathrm{mg} / \mathrm{day}$ of clopidogrel and $81 \mathrm{mg} / \mathrm{day}$ of aspirin for 10 days prior to the intervention. Platelet function tests were routinely performed using aspirin assay and P2Y12 assay (VerifyNow; Accumetrics) to obtain a level of platelet inhibition of 30\%-90\%. Patients with ruptured aneurysms underwent loading with $600 \mathrm{mg}$ of clopidogrel and $650 \mathrm{mg}$ of aspirin 8 hours prior to surgery. An initial heparin bolus of $100 \mathrm{U} / \mathrm{kg}$ was administered, and the activated clotting time was maintained at 2 times the patient's baseline intraoperatively. Heparin was discontinued at the end of the procedure. Dual antiplatelet therapy was continued for at least 6 months after the procedure.

Procedures were performed after induction of general endotracheal anesthesia and with continuous neurophysiological monitoring, including electroencephalography and somatosensory evoked potentials. The PEDs were delivered through a Marksman microcatheter (ev3), generally using a triaxial guide-catheter system. The number of stents inserted is left to the operator's discretion, but recently we have been using only a single device for most cases. The expansion of the PED was documented under fluoroscopy or with additional DynaCT/Xpert CT angiography at the operator's discretion. Angiographic follow-up (digital subtraction angiography or MR angiography) was scheduled at 3-6 months, 1 year, 2 years, and 5 years after treatment.

\section{Data Sources and Variables}

Medical charts were reviewed to collect patient age, sex, aneurysm size, aneurysm location, mode of presentation, and procedure details. Treatment success, procedural complications, clinical outcome, and initial and follow-up angiographic results were determined. Procedural success was defined as the successful placement of the PED at the targeted landing zone. All procedural complications (early and delayed) are reported regardless of their clinical significance. Aneurysm occlusion on follow-up angiograms was categorized as complete $(100 \%)$ or incomplete $(<100 \%)$.

\section{Statistical Analysis}

Data are presented as the median or mean and range for continuous variables, and as frequency and percent for categorical variables. Statistical analyses of categorical variables were performed using the chi-square, Fisher exact, and Mantel-Haenszel tests for linear association as appropriate. Statistics of means were calculated using the unpaired Student t-test and Wilcoxon rank-sum tests when variables were not normally distributed. The following dependent variables were assessed in univariate and multivariate analysis: complications, aneurysm obliteration, and retreatment. Kaplan-Meier risk of retreatment was calculated. Factors predictive of either obliteration or retreatment $(\mathrm{p}<0.2)$ were entered into multivariate Cox regression analysis to assess hazard ratios. Clinically significant variables and interaction expansion covariates were further assessed in Cox multivariate analysis as deemed relevant. Missing data points were excluded from the analysis. Probability values of $\leq 0.05$ were considered statistically significant.

\section{Results \\ Descriptive Data}

One hundred patients harboring small aneurysms, including 89 women and 11 men ranging in age from 17 to 80 years, underwent PED treatment. The mean aneurysm size was $5.2 \pm 1.5 \mathrm{~mm}$. Seven patients $(7 \% ; 5$ of them with blister aneurysms) had sustained a subarachnoid hemorrhage (SAH). All except 5 aneurysms (95\%) arose from the anterior circulation (Table 1). Morphologically, 11 aneurysms (11\%) were fusiform and $89(89 \%)$ were saccular. Twenty patients $(20 \%)$ had undergone previous surgical or endovascular treatment. Thirty-seven patients (37\%) were active smokers, 35 patients (35\%) had hypertension, 19 patients $(19 \%)$ had a family history of intracranial aneurysms, 40 patients $(40 \%)$ were $\leq 50$ years old, and 57 patients $(57 \%)$ had symptomatic aneurysms or reported headaches.

\section{Outcome Data and Main Results}

A total of 124 PEDs were inserted. Device placement was successful in all cases. Only 1 device was $\leq 3 \mathrm{~mm}$ in diameter. The number of PEDs used was 1.2 per aneurysm (range 1-4 PEDs). A single PED was used in 80 aneurysms (80\%), 2 PEDs were used in 17 aneurysms (17\%), 3 PEDs in 2 aneurysms (2\%), and 4 PEDs in 1 aneurysm (1\%). The

TABLE 1. Aneurysm locations and use of the PED in 100 patients

\begin{tabular}{lc}
\hline \multicolumn{1}{c}{ Aneurysm Location } & No. of PEDs $(\%)$ \\
\hline Carotid ophthalmic & $48(48)$ \\
\hline Carotid cavernous & $4(4)$ \\
\hline Vertebrobasilar & $5(5)$ \\
\hline Paraclinoid & $35(35)$ \\
\hline MCA & $3(3)$ \\
\hline Posterior communicating artery & $5(5)$ \\
\hline Total & 100 \\
\hline
\end{tabular}


mean number of devices used per lesion was 1.8 for fusiform aneurysms versus 1.1 for all remaining aneurysms ( $\mathrm{p}<0.001)$. In all ruptured aneurysms a single PED was used. Two aneurysms (2\%) were treated with adjunctive coils in addition to PED. Five patients (5\%) required balloon angioplasty because of inadequate PED expansion.

Symptomatic procedure-related complications occurred in 3 patients (3\%): 1 distal parenchymal hemorrhage managed conservatively (modified Rankin Scale [mRS] Score 2 at follow-up) and 2 ischemic events (mRS Score 0 and 1 , respectively, at follow-up). No complication occurred in patients with posterior circulation, ruptured, or fusiform aneurysms. None of the patients with SAH required an invasive procedure after PED placement (tracheostomy, feeding tube, ventriculostomy, ventriculoperitoneal shunt). No delayed aneurysm rupture occurred in the series. The following factors were tested for as predictors of complications: age, sex, aneurysm location, aneurysm size, aneurysm morphology, previous aneurysm treatment, number of PEDs used per aneurysm, adjunctive use of coils, and balloon angioplasty. There were no predictors of complications in univariate and multivariate analysis. Clinical follow-up was available for 96 patients at a mean of 7.3 months. All patients had achieved a favorable outcome (mRS Score 0-2) at the latest follow-up.

Angiographic follow-up was available for 75 patients $(75 \%)$ at a mean of 6.3 months. At the latest follow-up, 54 (72\%) of the 75 aneurysms with angiographic follow-up were completely occluded (100\%), $10(13 \%)$ were nearly completely occluded $(\geq 90 \%)$, and $11(15 \%)$ were incompletely occluded $(<90 \%)$. Accordingly, adequate aneurysm occlusion (complete or near-complete occlusion) was noted in $85 \%$ of cases. The rate of complete aneurysm occlusion was $83 \%$ (5 of 6 ) for ruptured aneurysms and $75 \%$ (6 of 8) for fusiform aneurysms. Six aneurysms (8\%) required further treatment. Actuarial rates of progression with no further treatment at 6 and 12 months were $98 \%$ and $90 \%$, respectively. The following factors were investigated as predictors of aneurysm obliteration (100\% occlusion) and retreatment: age, sex, aneurysm location, aneurysm size, aneurysm morphology, previous aneurysm treatment, number of PEDs used per aneurysm, adjunctive use of coils, balloon angioplasty, procedural complications, and angiographic follow-up time. In univariate analysis, older age $(\mathrm{p}=0.08)$ and increasing aneurysm size $(\mathrm{p}=0.12)$ predicted aneurysm occlusion. In multivariate analysis, there were no significant predictors for aneurysm occlusion. Increasing aneurysm size predicted retreatment in both univariate $(\mathrm{p}=0.05)$ and multivariate (OR 3.8, 95\% CI 0.99-14; $\mathrm{p}=0.05$ ) analysis.

\section{Discussion}

Flow diverters were initially reserved for complex, giant, and fusiform aneurysms. With the growing data supporting their safety and efficacy, flow diverters are now increasingly used in the management of small and less complex aneurysms at many institutions. Some, however, remain wary of flow diverters, especially for small aneurysms, and continue to prefer the traditional endovascular strategies-namely coiling and stent-assisted coiling. In
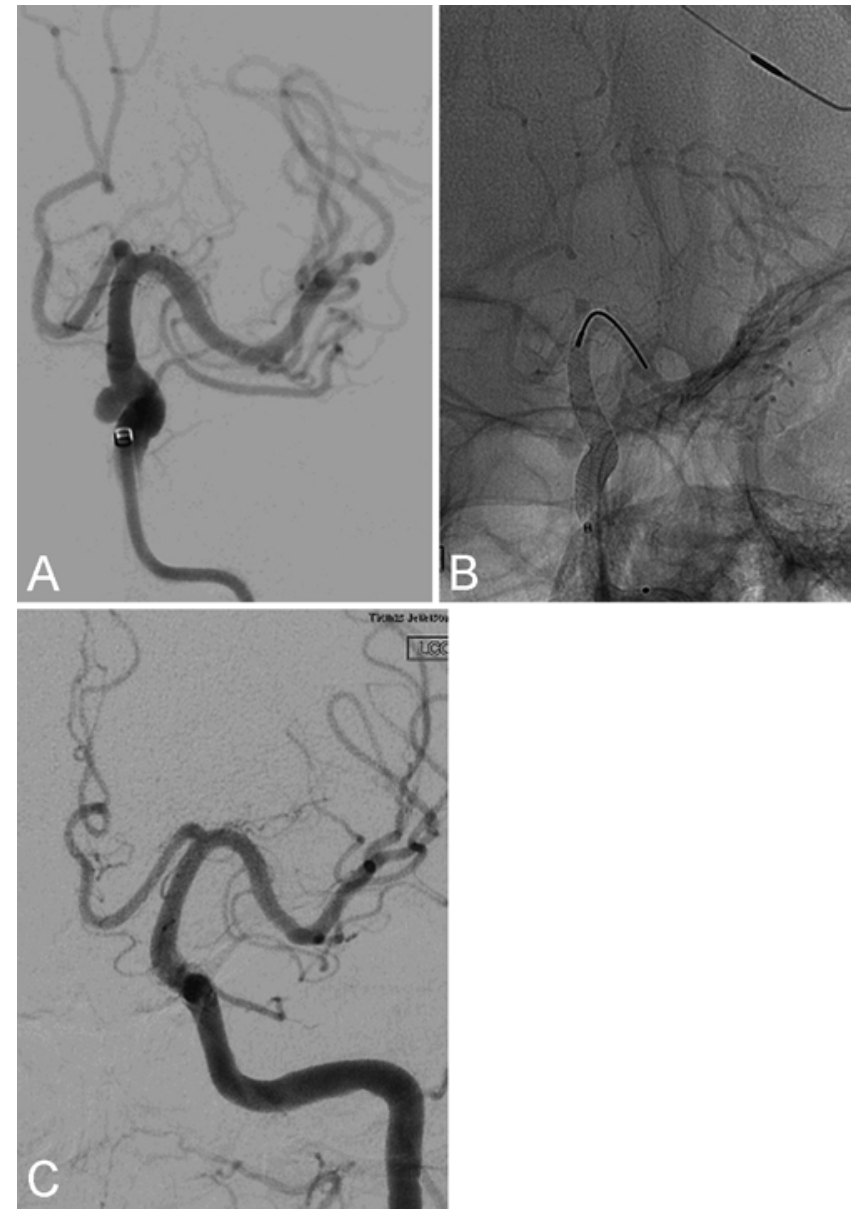

FIG. 1. Angiogram showing a small 5-mm aneurysm arising from the left ICA (A). A PED was inserted in the ICA (B), with complete occlusion at follow-up (C).

fact, for aneurysms $\leq 7 \mathrm{~mm}$, coiling has classically generated favorable results, ${ }^{3}$ whereas flow diversion remains experimental and has yet to gain FDA approval. In the current study we aimed to determine in a large series of patients whether treatment of small aneurysms with the PED is safe and effective (Figs. 1 and 2). We found that PED treatment can be undertaken with a complication rate as low as 3\%, and is associated with excellent clinical outcomes. Importantly, the occlusion rate with the PED was as high as $85 \%$, which confirms the high efficacy of the device in this subgroup of patients. These findings suggest that it is safe to offer PED therapy as an option for patients with aneurysms $<7 \mathrm{~mm}$ in whom treatment is contemplated.

The findings of this study add to the results of the published literature on the PED. We are aware of only 1 study that specifically assessed the safety and efficacy of the PED in small aneurysms. In this study, which included 41 patients with 44 aneurysms $<10 \mathrm{~mm}$ (vs $\leq 7 \mathrm{~mm}$ in our study), Lin et al..$^{15}$ reported major complications in only 1 case $(2.3 \%)$, with an occlusion rate as high as $91.8 \%$ after a mean follow-up of 4 months. All patients in the study remained at their neurological baseline at the latest followup. In a large Turkish series of 191 patients treated with the PED, 155 aneurysms were $<10 \mathrm{~mm}$, but the authors 

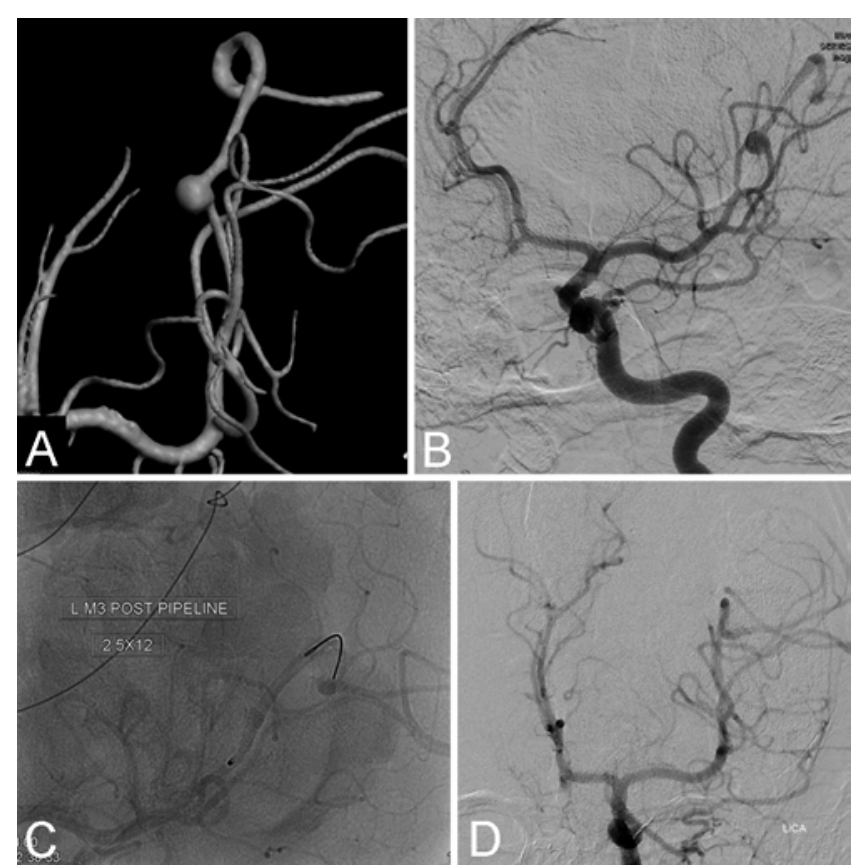

FIG. 2. Angiogram showing a 5-mm fusiform aneurysm arising from the $\mathrm{M}_{3}$ segment of the middle cerebral artery (MCA) (A and B). A PED was successfully placed across the aneurysm in the MCA (C). Follow-up angiogram showing $100 \%$ aneurysm occlusion, with remodeling of the parent vessel (D).

did not report how many were $<7 \mathrm{~mm}$. The 6-month occlusion rate was $94 \%$ for aneurysms $<10 \mathrm{~mm}$ and the permanent morbidity rate was as low as $1 \% .^{19}$ Taken together, these data support the favorable safety-efficacy profile of the PED in small aneurysms. This replicates the findings of PED therapy in large and giant aneurysms. In the PUFS (Pipeline for Uncoilable or Failed Aneurysms) trial, which only included large and giant aneurysms, the occlusion rate was $74 \%$ and the major stroke or neurological death rate was only $5.6 \% .^{1}$ In a case-controlled study, Chalouhi et al. ${ }^{5}$ found that PED treatment provided higher occlusion rates for large and giant aneurysms than coiling, with a similar complication rate, and concluded that PED is the preferred option for those aneurysms.

Despite the favorable results achieved in the present and previous reports, neurosurgeons should be aware that flow diversion remains somewhat in its infancy; long-term safety data are lacking. It is important to note that treatment of aneurysms $\leq 7 \mathrm{~mm}$ with the PED is an off-label use of the device. Also, flow diverters are associated with some risks that are not encountered with conventional endovascular techniques-namely distal parenchymal hemorrhage, delayed migration of the device, and delayed aneurysm rupture. ${ }^{6,12,16}$ Distal parenchymal hemorrhage may occur ipsilaterally or even contralaterally to the aneurysm, and its mechanism is thought to involve hemorrhagic conversion of ischemic lesions in the setting of dual antiplatelet therapy, embolized foreign material, or loss of autoregulation of the distal arteries. ${ }^{9,17}$ Delayed aneurysm rupture after flow diversion occurs in approximately $1 \%$ of patients, and its mechanism may involve sudden hemodynamic changes in intraaneurysmal flow pattern or aneu- rysm wall weakening due to inflammation and proteolytic enzymes within the developing red thrombus. ${ }^{17}$ Some have suggested using intraluminal coils or steroids to attenuate the effects of intraaneurysmal thrombosis, but it remains to be seen whether this strategy can reduce the risk of aneurysm rupture. Last, device migration has recently been recognized as a potential severe complication of flow diversion that can be associated with hemorrhagic or thromboembolic events. ${ }^{4}$ Migration may occur proximally or distally and is related to the mismatch in arterial diameter between inflow and outflow vessels, and to stretching of the device. ${ }^{11}$ Fortunately, these complications appear to be uncommon in small aneurysms, as suggested by the present report.

\section{Limitations of the Study}

The main limitations of this study are related to its retrospective design, absence of a control group, and absence of long-term safety and efficacy data. It is important to determine the long-term risk of thromboembolism with and without clopidogrel as well as the long-term protection provided by subtotal aneurysm occlusion with flow diverters. It is known that clipping and coiling have classically generated excellent results in small aneurysms, and so it remains to be seen whether flow diversion provides superior results. This study also reflects the experience of a single high-volume center, and therefore results may not be completely generalizable to other centers. Despite these limitations, this study shows the feasibility, safety, and efficacy of the PED in aneurysms that are usually not typically treated with this approach, and it serves to allay some of the concerns about extending the indications of flow diverters to other types of aneurysms. Some may argue against treating aneurysms $\leq 7 \mathrm{~mm}$ given their favorable natural history, with an annual risk of rupture < $0.5 \% .^{2,10}$ Although aneurysms $\leq 7 \mathrm{~mm}$ have a lower risk of rupture than larger aneurysms, it is our practice to treat such patients in the presence of risk factors such as personal or family history of SAH, smoking, hypertension, daughter sac, young age, symptomatic aneurysm, multilobed aneurysm, and aneurysm progression. ${ }^{2}$ Treated patients represent only a small percentage of individuals with aneurysms seen in our practice, in most of whom the lesions are managed conservatively.

\section{Conclusions}

In this study, treatment of small aneurysms with the PED was associated with low complication rates and high aneurysm occlusion rates. These findings suggest that the PED is a safe and effective alternative to conventional endovascular techniques for small aneurysms. Randomized trials with long-term follow-up are necessary to determine the optimal treatment that leads to the highest rate of obliteration and the best clinical outcomes.

\section{References}

1. Becske T, Kallmes DF, Saatci I, McDougall CG, Szikora I, Lanzino G, et al: Pipeline for uncoilable or failed aneurysms: results from a multicenter clinical trial. Radiology 267:858868,2013 
2. Chalouhi N, Dumont AS, Randazzo C, Tjoumakaris S, Gonzalez LF, Rosenwasser R, et al: Management of incidentally discovered intracranial vascular abnormalities. Neurosurg Focus 31(6):E1, 2011

3. Chalouhi N, Jabbour P, Singhal S, Drueding R, Starke RM, Dalyai RT, et al: Stent-assisted coiling of intracranial aneurysms: predictors of complications, recanalization, and outcome in 508 cases. Stroke 44:1348-1353, 2013

4. Chalouhi N, Satti SR, Tjoumakaris S, Dumont AS, Gonzalez LF, Rosenwasser R, et al: Delayed migration of a Pipeline Embolization Device. Neurosurgery 72 (2 Suppl Operative):ons229-ons234, 2013

5. Chalouhi N, Tjoumakaris S, Starke RM, Gonzalez LF, Randazzo C, Hasan D, et al: Comparison of flow diversion and coiling in large unruptured intracranial saccular aneurysms. Stroke 44:2150-2154, 2013

6. Chalouhi N, Tjoumakaris SI, Gonzalez LF, Hasan D, Pema PJ, Gould G, et al: Spontaneous delayed migration/shortening of the pipeline embolization device: report of 5 cases. AJNR Am J Neuroradiol 34:2326-2330, 2013

7. D'Urso PI, Lanzino G, Cloft HJ, Kallmes DF: Flow diversion for intracranial aneurysms: a review. Stroke 42:2363-2368, 2011

8. De Vries J, Boogaarts J, Van Norden A, Wakhloo AK: New generation of Flow Diverter (surpass) for unruptured intracranial aneurysms: a prospective single-center study in 37 patients. Stroke 44:1567-1577, 2013

9. Deshmukh V, Hu YC, McDougall CG, Barnwell SL, Albuquerque F, Fiorella D: Histopathological assessment of delayed ipsilateral parenchymal hemorrhages after the treatment of paraclinoid aneurysms with the Pipeline Embolization Device. Neurosurgery 71:E551-E552, 2012

10. Morita A, Kirino T, Hashi K, Aoki N, Fukuhara S, Hashimoto N, et al: The natural course of unruptured cerebral aneurysms in a Japanese cohort. N Engl J Med 366:2474-2482, 2012

11. Jabbour P, Chalouhi N, Tjoumakaris S, Gonzalez LF, Dumont AS, Randazzo C, et al: The Pipeline Embolization Device: learning curve and predictors of complications and aneurysm obliteration. Neurosurgery 73:113-120, 2013

12. Jabbour PM, Chalouhi N, Rosenwasser RH: The Pipeline Embolization Device: what have we learned? World Neurosurg 80:798-799, 2013

13. Kallmes DF, Ding YH, Dai D, Kadirvel R, Lewis DA, Cloft HJ: A new endoluminal, flow-disrupting device for treatment of saccular aneurysms. Stroke 38:2346-2352, 2007
14. Kan P, Siddiqui AH, Veznedaroglu E, Liebman KM, Binning MJ, Dumont TM, et al: Early postmarket results after treatment of intracranial aneurysms with the pipeline embolization device: a U.S. multicenter experience. Neurosurgery 71:1080-1088, 2012

15. Lin LM, Colby GP, Kim JE, Huang J, Tamargo RJ, Coon AL: Immediate and follow-up results for 44 consecutive cases of small $(<10 \mathrm{~mm})$ internal carotid artery aneurysms treated with the pipeline embolization device. Surg Neurol Int 4:114, 2013

16. Piano M, Valvassori L, Quilici L, Pero G, Boccardi E: Midterm and long-term follow-up of cerebral aneurysms treated with flow diverter devices: a single-center experience. J Neurosurg 118:408-416, 2013

17. Pierot L, Wakhloo AK: Endovascular treatment of intracranial aneurysms: current status. Stroke 44:2046-2054, 2013

18. Pistocchi S, Blanc R, Bartolini B, Piotin M: Flow diverters at and beyond the level of the circle of Willis for the treatment of intracranial aneurysms. Stroke 43:1032-1038, 2012

19. Saatci I, Yavuz K, Ozer C, Geyik S, Cekirge HS: Treatment of intracranial aneurysms using the Pipeline Flow-Diverter Embolization Device: a single-center experience with longterm follow-up results. AJNR Am J Neuroradiol 33:14361446, 2012

20. Yu SC, Kwok CK, Cheng PW, Chan KY, Lau SS, Lui WM, et al: Intracranial aneurysms: midterm outcome of Pipeline Embolization Device - a prospective study in 143 patients with 178 aneurysms. Radiology 265:893-901, 2012

\section{Author Contributions}

Conception and design: Jabbour, Chalouhi, Zanaty, Tjoumakaris, Kung, Rosenwasser. Acquisition of data: Whiting, Yang, Hann, Hammer. Analysis and interpretation of data: Chalouhi, Zanaty, Whiting, Hasan, Starke. Drafting the article: Chalouhi, Yang, Starke. Critically revising the article: Jabbour, Chalouhi, Zanaty, Whiting. Reviewed submitted version of manuscript: all authors. Approved the final version of the manuscript on behalf of all authors: Jabbour. Statistical analysis: Hasan. Study supervision: Jabbour, Rosenwasser.

\section{Correspondence}

Pascal M. Jabbour, Division of Neurovascular Surgery and Endovascular Neurosurgery, Department of Neurological Surgery, Thomas Jefferson University Hospital, 901 Walnut St., 3rd Fl., Philadelphia, PA 19107. email: pascal.jabbour@jefferson.edu. 